\title{
In-situ Synthesis and in-operando Swelling/ Deswelling of PolyNIPAM Nanoparticles
}

Wyeth Gibson and Joesph Patterson

Univeristy of California - Irvine, Irvine, California, United States

Poly(N-isopropylacrylamide) (pNIPAM) is a functional material that has been of interest due to its temperature responsive properties. ${ }^{1}$ On an industrial scale pNIPAM is synthesized using both chemical and electron beam initiated processes. ${ }^{2-4}$ Although the chemistries are well known, there have been no investigation into the mechanistic difference between the chemical and electron beam initiated processes. Consequently, we believe this is an ideal opportunity to utilize liquid phase transmission electron microscopy (LPTEM) to study the formation of pNIPAM close to in-operando. Comparing the results to particles synthesized chemically in the lab and analyzed by cryoTEM we aim to elucidate the mechanistic differences between the two synthetic processes. A key challenge for these experiments is that the electron fluxes used in LPTEM are significantly higher than the fluxes used in the industrial electron beam initiated processes.

pNIPAM has a drastic change in its interaction with an aqueous solvent at biologically relevant temperatures $\left(\approx 32^{\circ} \mathrm{C}\right)$. This change is due to the free energy of the polymer-water interface increasing and a polymer-rich phase is produced, the temperature this occurs at is referred to as the cloud point. This polymer and solvent demixing results from the individual polymer changing structure from a coil to a globular structure due to interactions with itself becoming lower in free energy compared to interactions with water. ${ }^{1}$ All of this manifests itself as a precipitation of the polymer from solution or the expansion and contraction of the polymer from a surface it is attached to. Numerous lines of research have resulted, with many directed toward its use in biological systems. A current use is pNIPAM coated petri dishes that increase the efficacy of mammalian cell culture methods. ${ }^{5}$ During cell growth the dish is kept above its cloud point causing the polymer structure to be compressed against the petri dish surface. When cells are to be harvested, the dish is cooled to below the cloud point and the polymer expands, aiding in the nondestructive release of cells from the petri dish. These are produced by exposing the plates containing a solution of NIPAM monomer and isopropanol to an electron beam which polymerizes and grafts the polymer to the surface. In our experiments we expose a solution of water and NIPAM to the electron beam in the TEM as we image producing nanoparticles (Figure 1).

In many LPTEM experiments attention is paid to how beam-sample interactions are manifesting in the experiment. Of large concern is the production of reactive species that are not present in the bench top chemistry. ${ }^{6}$ In this experiment we approach the bench top conditions due to the fact that the industrial process utilizes electron beam polymerization techniques to graph the polymers on to surfaces. Differences do exist in the solvent used (isopropanol versus water) and the electrons the sample is experiencing (potential and flux). Our goal is to not only address these differences but also establish how these along with temperature dictate the product of these e-beam polymerizations. Isopropanol and other alcohols have been shown to scavenge radicals in the electron beam environment which most likely means the population and type of radicals produced in the water system is different. These reactive species can change the properties of the particles formed. It has been shown that pNIPAM can be crosslinked in absence of a crosslinking agent. ${ }^{7}$ So, it could be predicted that the amount of radical scavenger such as isopropanol can determine the extent of crosslinking in the nanoparticle. Determining the differences in 
flux and potential can be assessed by comparing products of the TEM experiments versus the product of sample submitted to an industrial electron beam processing facility. Using water in conjunction with a temperature-controlled liquid cell, we can probe the relationship between pNIPAM's thermal responsive properties and the formation of the e-beam induced particle formation. We have shown in experiments that final size and morphology can be dictated by the temperature during synthesis (Figure 2). Particularly at temperatures above and below the cloud point. A characteristic that we believe to not exist in isopropanol as a cloud point doesn't exist for pNIPAM in it.

In the presentation I will show the observations offered by LPTEM imaging the evolution of pNIPAM particle generation initiated by an electron beam. These observations approach in operando conditions which have the potential of offering scalable insights to large scale production. Differences between the experimental conditions in the LPTEM holder and industrial processes will also be addressed.

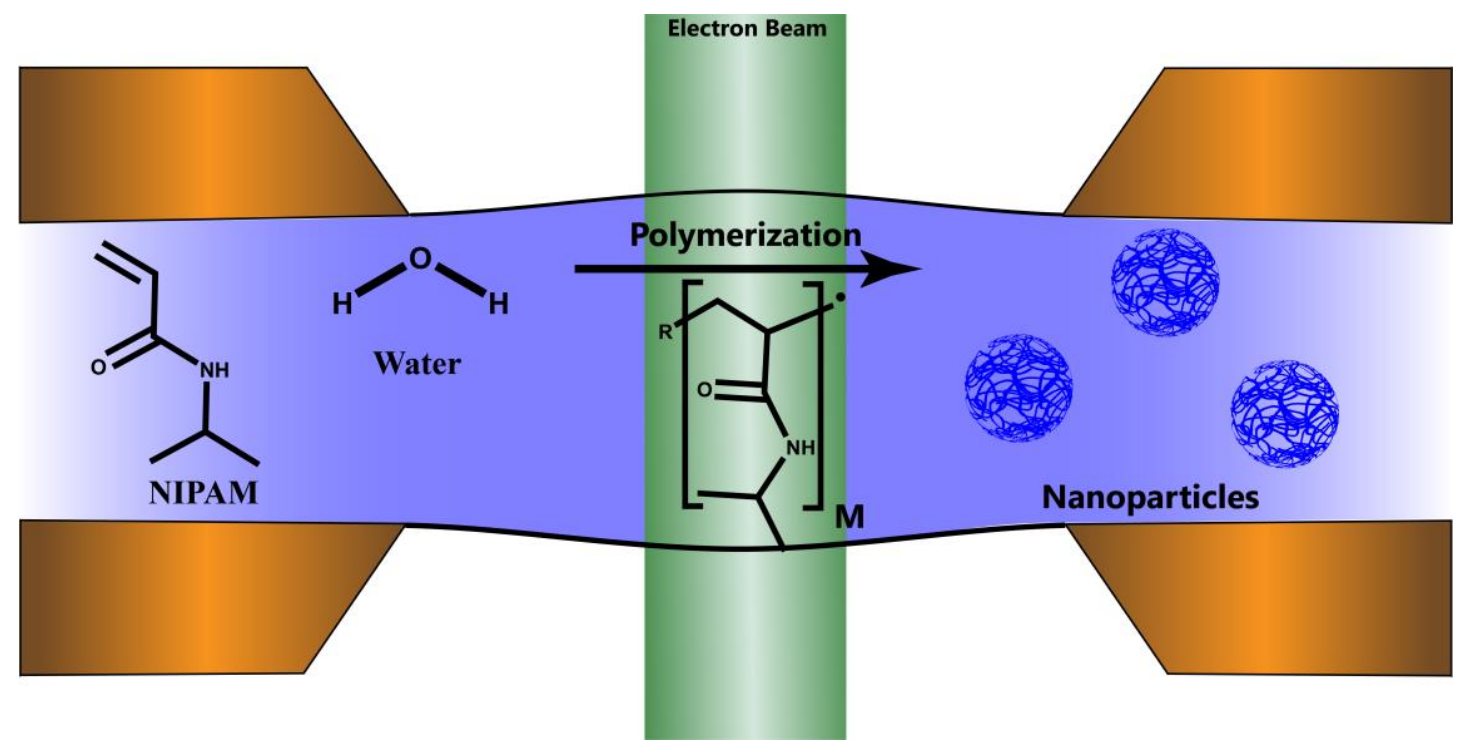

Figure 1. Figure 1 Depiction of our experimental setup showing the dissolved NIPAM monomer going through electron beam polymerization and producing nanoparticles.

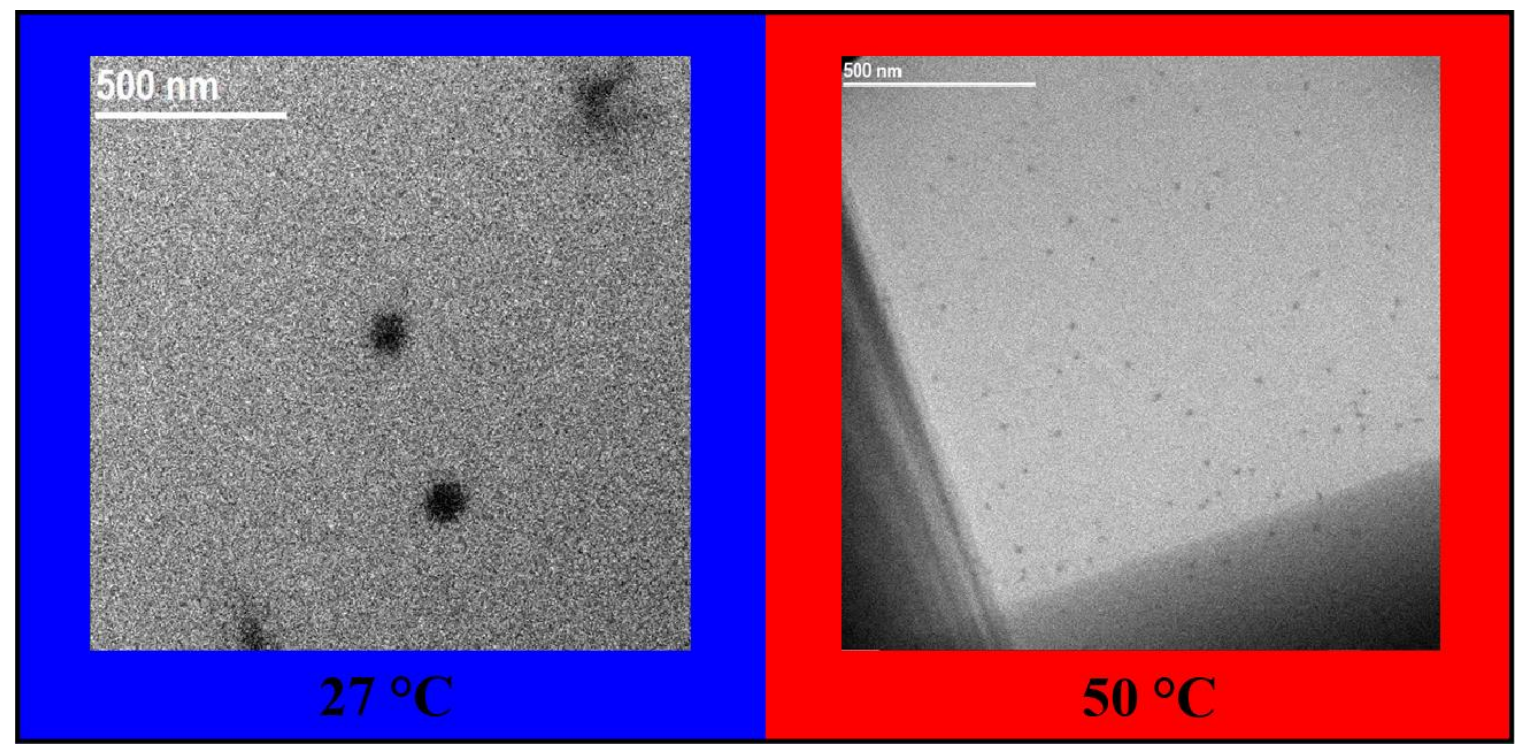


Figure 2. Figure 2 A) In situ liquid phase TEM image sampled from a four-minute movie showing the formation of pNIPAM nanoparticles at $27{ }^{\circ} \mathrm{C}$ with two different morphologies (spheres and branched particles). B) In situ liquid phase TEM sample image showing formation of nanoparticles at $50{ }^{\circ} \mathrm{C}$ with a closer to monodisperse spherical morphology.

References

1. Halperin, A.; Kröger, M.; Winnik, F. M., Poly(N-isopropylacrylamide) Phase Diagrams: Fifty Years of Research. Angewandte Chemie International Edition 2015, 54 (51), 15342-15367.

2. Akiyama, Y.; Kikuchi, A.; Yamato, M.; Okano, T., Ultrathin Poly(N-isopropylacrylamide) Grafted Layer on Polystyrene Surfaces for Cell Adhesion/Detachment Control. Langmuir 2004, 20 (13), 55065511.

3. Lanzalaco, S.; Armelin, E., Poly(N-isopropylacrylamide) and Copolymers: A Review on Recent Progresses in Biomedical Applications. Gels 2017, 3 (4), 36.

4. Gangadhar Panambur, I. K., and Scott Batcheller, Designing temperature and $\mathrm{pH}$ sensitive NIPAM based polymers. MillporeSigma White Paper.

5. Cell Harvesting by Temperature Reduction. Thermo Fisher Scientific White Paper.

6. Schneider, N. M.; Norton, M. M.; Mendel, B. J.; Grogan, J. M.; Ross, F. M.; Bau, H. H., ElectronWater Interactions and Implications for Liquid Cell Electron Microscopy. The Journal of Physical Chemistry C 2014, 118 (38), 22373-22382.

7. Brugnoni, M.; Nickel, A. C.; Kröger, L. C.; Scotti, A.; Pich, A.; Leonhard, K.; Richtering, W., Synthesis and structure of deuterated ultra-low cross-linked poly(N-isopropylacrylamide) microgels. Polymer Chemistry 2019, 10 (19), 2397-2405. 